\title{
Resolution improvement of optical coherence tomography with software spectral shaping
}

\author{
Chih-Wei Lu, Meng-Tsan Tsai, Yih-Ming Wang, Yean-Woei Kiang and C. C. Yang \\ Graduate Institute of Electro-Optical Engineering and Department of Electrical Engineering, \\ National Taiwan University, 1, Roosevelt Road, Section 4, Taipei, Taiwan \\ (phone) 886-2-23657624 (fax) 886-2-23652637 (E-mail) ccy@.cc.ee.ntu.edu.tw
}

Abstract -- We demonstrate dynamic spectral shaping in an optical coherence tomography (OCT) system with multiple scans of segmented spectral bands and software signal processing. With an effective spectral full-width at half-maximum ( $F W H M$ ) of $45 \mathrm{~nm}$, centered at $950 \mathrm{~nm}$, we compress the interference fringe FWHM from 31 microns down to 7 microns, which is smaller than the theoretical limit of 8.9 microns if a Gaussian spectral shape is assumed, through the combined effect of dispersion compensation and spectral shaping.

\section{INTRODUCTION}

Besides the use of broadband light sources for achieving high resolution, other efforts are helpful for improving the OCT resolution, including dispersion compensation for balancing the group delay dispersion between the reference and sample arms of an OCT system. The other means for improving resolution is the spectral shaping technique. Spectral shaping means to modify the spectral intensity and phase distributions for controlling the time-domain behaviors. It has been widely used for modifying the pulse shape and width [1-3]. This technique was also used for changing the width and shape of the interference fringe pattern of an OCT system. It is particularly useful when the light source spectrum is not Gaussian-like. With a non-Gaussian-like spectral profile, side-lobes in the interference fringe pattern exist. Such side-lobes will degrade the OCT resolution. A software approach, based on an iteration retrieval algorithm, has been proposed to reduce the side-lobes [4]. Also, spectral shaping effects were obtained from the spectrally filtered response of each depth profile by multiplying the Fourier transform of each individual depth profile with a spectral correction function and performing the inverse transform [5]. Spectral shaping is quite a promising technique for improving the OCT resolution. In this paper, we report a novel technique for dynamic spectral shaping. In this technique, OCT images are first obtained with segmented light source spectra. Then, an appropriate combination of these images of different central wavelengths can result in various results of either higher axial resolution or reduced side-lobe intensity. Such a combination can be dynamically adjusted for different purposes. Also, it can be used for dispersion compensation.

\section{EXPERIMENTAL PROCEDURES}

An OCT system was built with the light source of $950 \mathrm{~nm}$ in central wavelength and $45 \mathrm{~nm}$ in effective full-width at half-maximum (FWHM). Therefore, the theoretical axial resolution is 8.9 microns if the spectral shape is Gaussian. In the reference arm, an optical phase delay line (OPDL) was used for group- and phase-delay modulations. To match the optical paths between the reference and sample arms, an about $25 \mathrm{~cm}$ fiber length difference is required. In the proposed approach, we used a spatial filter, located between the lens and the galvanometer, for gating different wavelength bands of the light source spectrum. We conducted a longitudinal scan with each spectral band. Due to dispersion, the interference fringes of different spectral bands will appear at different longitudinal positions. After properly adjusting the positions and amplitudes of the scanning images of various segmented spectral bands, their superposition leads to the results of various spectral shaping effects. The FWHM of the interference fringe envelope was 31 microns with dispersion. The spectral segmentation is implemented by translating the $1-\mathrm{mm}$ wide spatial filter in the transverse direction to the optical path. Six segmented spectral bands and hence six depth scans were obtained by translating the spatial filter with 700 microns in step size. The central wavelengths of the six bands are located at 930 through $980 \mathrm{~nm}$ with 10 $\mathrm{nm}$ in step size. Each has a FWHM of about $15 \mathrm{~nm}$ and a super-Gaussian shape.

\section{EXPERIMENTAL RESULTS}

In Fig. 1, the interference fringe patterns of the six longitudinal scans of a glass surface with the six successive segmented spectral bands are shown. Parts (a)-(f) correspond to the results from the short to the long central wavelength. Here, one can see that the six fringe patterns are located at slightly different positions due to dispersion. The FWHMs of those interference fringe envelopes are all about 27 microns, which is close to the theoretical limit of the $15 \mathrm{~nm}$ FWHM in a segmented spectral band. This result shows that the dispersion effect is negligible in such a small bandwidth. Note that the modulation frequencies of the six interference fringes are also different because of their different central wavelengths. 


\section{THP-5(T1)}

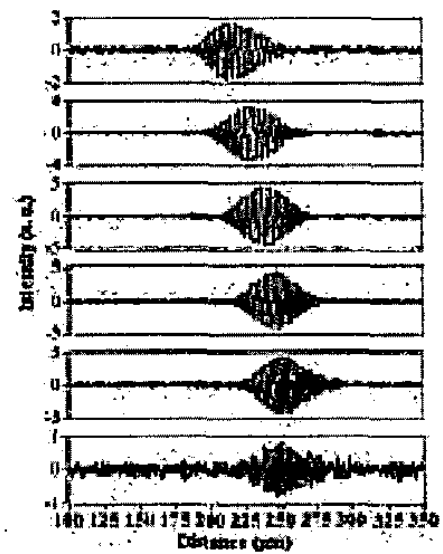

Fig. 1 Six interference fringes with different segmented spectral bands.

After shifting the positions of the six interference-fringe patterns to align their envelope maxima in the horizontal axis in which the dispersion mismatch has been significantly compensated. The envelope FWHM is now reduced to 7 microns, as shown in Fig. 2. Note that significant side-lobes exist in Fig. 2 with the intensity up to $22.6 \%$ of the main peak. The OCT resolution of 7 microns is higher than the theoretical limit ( 8.9 microns) if the spectral shape is Gaussian. The smaller envelope FWHM can be attributed to the larger effective full spectral width, which can be obtained by taking the summation of the six segmented spectral bands. In other words, spectral shaping has been effectively implemented. The reduced envelope FWHM was obtained at the expense of significant side-lobes.

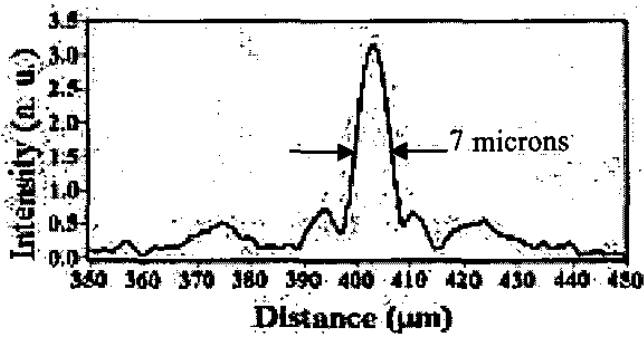

Fig. 2 Interference fringe envelope (reduced to 7 microns) after the software process.

\section{DISCUSSIONS AND CONCLUSIONS}

The proposed approach can also be used for dispersion compensation. The alignment of the envelope maxima of parts (a)-(f) in Fig. I before superposition means to compensate the dispersion mismatch between the reference and sample arms. In other words, the separations between the six interference fringes represent the group delay differences of the OCT system at various wavelengths. The result shown in Fig. 2 was obtained through the combined effects of dispersion compensation and spectral shaping. In summary, we have demonstrated dynamic spectral shaping in an OCT system with multiple scans of segmented spectral bands and software signal processing. With an effective spectral FWHM of $45 \mathrm{~nm}$, centered at $950 \mathrm{~nm}$, we compressed the interference fringe FWHM from 31 microns down to 7 microns, which is smaller than the theoretical limit of 8.9 microns if a Gaussian spectral shape is assumed, through the combined effect of dispersion compensation and spectral shaping. With the amplitude adjustments of the OCT images of the segmented spectral bands, side-lobes could be significantly suppressed with slight degradation of axial resolution. With other amplitude adjustments, the fringe-envelope FWHM was reduced to 5.6 microns at the expense of strong side-lobes.

\section{REFERENCES}

1. M. Takeoka, D. Fujishima, and F. Kannari, Opt. Lett. 26, 1592-1594 (2001).

2. A. M. Weiner and A. M. Kan'an, IEEE J. Select. Topics Quantum Electron. 4, 317-331 (1998).

3. R. Grote and H. Fouckhardt, Opt. Express 8, 328-335 (1999).

4. I. J. Hsu, C. W. Sun, C. W. Lu, C. C. Yang, C. P. Chiang, and C. W. Lin, Appl. Opt. 42, 227-234 (2003).

5. R. Tripathi, N. Nassif, J. S. Nelson, B. H. Park, J. F. de Boer, Opt. Lett. 27, 406-408 (2002). 